\title{
UV-spectrophotometry determination of taurine in energy drink mixtures
}

\author{
Georgi B. Draganov, Ivanka P. Pencheva, Katerina A. Todorova \\ Dept. of Pharmaceutical chemistry, Faculty of Pharmacy, Medical University - Sofia, Sofia, Bulgaria \\ Email address: \\ ivatibi@gmail.com (I. P. Pencheva)
}

To cite this article:

Georgi B. Draganov, Ivanka P. Pencheva, Katerina A. Todorova. UV-Spectrophotometry Determination of Taurine in Energy Drink Mixtures. International Journal of Nutrition and Food Sciences. Vol. 3, No. 2, 2014, pp. 123-126. doi: 10.11648/j.ijnfs.20140302.26

\begin{abstract}
The aim of this study is developing and validation of UV-spectrophotometric method for determination of taurine in energy drink mixtures. The investigation includes validation of procedures for performance of the tests for identification, purity and assay. Analytical parameters precision, accuracy, selectivity, linearity, limit of detection and limit of quantitation were studied and compared. Developed method allows selectively determination of taurine in the present of caffeine at different conditions (algorithm, wavelength zones). The preferences are estimation the analytical parameters from the validation procedures. They let to compose the criteria in accordance with European Pharmacopoeia and EU regulates about the use of official analytical programs for quality control of supplements containing taurine. The method has been applied successfully in analysis of energy food drinks preparation in the appointed for each of their aspect of applications.
\end{abstract}

Keywords: Taurine, Energy Food Drinks, Ninhydrin Reaction, UV-VIS Spectrophotometry

\section{Introduction}

Taurine (2-aminoethanesulfonic acid) is an organic acid derivative of cysteine which has a regular usage in energy food drinks. There are written many physiology effects of taurine which are explained with amino terminal group in the structure and sulfonic acid group moiety $[1,2]$. Taurine crosses the blood-brain barrier and has been implicated in a wide array of physiological phenomena. It includes inhibitory neurotransmission, long-term potential in the striatum/hippocampus membrane stabilization, feedback inhibition of neutrophil/macrophage respiratory burst, adipose tissue regulation. Taurine takes part in the possible prevention of obesity, calcium homeostasis, recovery from osmotic shock, protection against glutamate citotoxicity and prevention of epileptic seizures. It also acts as an antioxidant and protects against toxicity of various substances (such as lead and cadmium). Additionally, supplementation with taurine has been shown to prevent oxidative stress induced by exercise. Taurine helps people with congestive heart failure by increasing the force and effectiveness of heart-muscle contractions. Studies have shown that taurine can influence defects in nerve blood flow, motor nerve conduction velocity, and nerve sensory thresholds in experimental diabetic neuropathic rats. Taurine is conjugated via its amino terminal group with chenodeoxycholic acid and cholic acid to form the bile salts sodium taurochenodeoxycholate and sodium taurocholate. The low pKa of taurine's sulfonic acid group ensures that this moiety is negatively charged in the $\mathrm{pH}$ ranges normally found in the intestinal tract and, thus, improves the surfactant properties of the cholic acid conjugate. Taurine is necessary for normal skeletal muscle functioning [2-4].

The wide-spread usage of energy drinks with taurine increases the cases with unwanted side effects and requires the development of an analytical program including identification tests and assays with accuracy, precision and selectivity for monitoring of number supplements especially for those which has free distribution on the market.

For determination of taurine several analytical methods are described: HPLC with pre- and post - column derivatization with different reagents and UV detection, HPLC with mass spectrometry; HILIC-chromatography with UV and ELSD detection, UV-spectrophotometric methods [5-18]. All this methods solve specific problems in rather different matrixes and for different aims.

Aim: The aim of this study is a developing and validation of UV-spectrophotometry method for identification and quantitation of taurine in supplement and drug mixtures with high selectivity and wide spectra of applications in the 
compliance with European Pharmacopoeia regulates.

\section{Experimental Methods}

\subsection{Reagents}

Taurine reference substance (RS), Caffeine RS, ninhydrin, 70 vol. \% ethanol, energy food drinks, containing $35 \mathrm{mg}$ taurine, model mixtures from reference substances, containing 25,35 and $50 \mathrm{mg}$ taurine and $150 \mathrm{mg}$ caffeine.

\subsection{System}

UV/VIS Spectrometer HP;

Diode array detector;

Wavelength Range - $190-820 \mathrm{~nm}$;

Wavelenght Accuracy $- \pm 2 \mathrm{~nm}$;

UV/VIS operating software.

\subsubsection{Analytical Calculations}

Analytical calculations are based on Single standard method at fixed conditions. Method's options are given on table 1 .

Table 1. Analytical calculations.

\begin{tabular}{ll}
\hline Calculations & Method's options \\
\hline Analysis & Single standard method (SCA) \\
Calibration Curve type & Beers Law \\
Algorithm & Least Squares fit (LSQ) \\
Derivative order & 0 \\
Polynomial Degree & 0 \\
Smoothing Points & 1 \\
Data Interval & $2 \mathrm{~nm}$ \\
Analytical Wavelength Zone & $200 \mathrm{~nm}$ to $800 \mathrm{~nm}$ \\
Temperature & $25^{\circ} \mathrm{C}$ \\
\hline
\end{tabular}

\subsection{Sample Preparation}

\subsubsection{Reference Solutions}

Reference solutions containing taurine and caffeine were prepared by dissolving of accurately weighed $35 \mathrm{mg}$ RS taurine and $50 \mathrm{mg}$ RS caffeine with $10 \mathrm{ml} 70$ vol. \% ethanol. Aliquot volumes from solutions were diluted with the same solvent to obtain solutions with appropriate known concentration.

\subsubsection{Test Solutions}

Model mixtures containing $35 \mathrm{mg}$ taurine RS and $50 \mathrm{mg}$ caffeine RS were dissolved with 70 vol. \% ethanol in volumetric flask up to $10 \mathrm{ml} .1 .0 \mathrm{ml}$ from obtained solution was diluted to obtain solutions with appropriate concentration.

Test solutions of model mixtures containing 25,35 and 50 $\mathrm{mg}$ taurine RS and $150 \mathrm{mg}$ caffeine RS were prepared by the same manner.

Aliquot volume from energy food drink containing $35 \mathrm{mg}$ taurine and $50 \mathrm{mg}$ caffeine was evaporated to dryness and the residue was dissolved with $10 \mathrm{ml} 70$ vol. \% ethanol and diluted with the same solvent to obtain a solution with necessary concentration.

\subsubsection{Blank Solution}

70 vol. \% ethanol.

\subsubsection{Procedure}

$5.0 \mathrm{ml}$ from each of reference, test and blank solutions described above were heated with $5.0 \mathrm{ml} 0.2 \%$ solution of ninhydrine for $20 \mathrm{~min}$ at $70{ }^{\circ} \mathrm{C}$. After cooling the obtained sample solutions were diluted up to necessary volume.

The prepared solutions were analyzed by normal spectrophotometry in VIS range at fixed wavelength $570 \mathrm{~nm}$ for model mixtures containing taurine-ninhydrin color compound against 70 vol. \% ethanol matrix as blank solution. The investigations were carried out using single standard method.

\section{Results and Discussion}

In energy drink mixtures taurine usually is in concomitant composition with xanthine derivative caffeine which quantities vary from 50 to $150 \mathrm{mg}$. Caffeine shows intensive and specific absorption in UV range and at simultaneously UV-spectrophotometry determination with taurine it's UV spectra cover those of taurine in analytical zone from 190 to $400 \mathrm{~nm}$ (fig. 2, (1)). Due to this authors take advantage to the fact that taurine reacts selectively with ninhydrin after warming. The product of reaction is a color compound and it has absorption max at $570 \mathrm{~nm}$ in 70 vol. \% ethanol matrix (fig. 2, (2)). Ninhydrin reacts with primary and secondary amines producing a blue or purple reaction product (diketohydrindylidene - diketohydrindamine)[19](fig. 1). The intensity of coloring is proportional to concentration of analyzed compounds (first order reaction).

The reaction was negative to caffeine, tertiary amines and amines with aromatic moiety.

Based on it UV-spectrophotometry method was developed and validated in respect of analytical parameters selectivity, precision, accuracy, linearity, limit of detection and limit of quantitation. The conditions were verified for taurine as reference substance and energy mixtures with caffeine with negative ninhydrin test.<smiles>O=C1C(=O)c2ccccc2C(O)=C1N=c1c(=O)c2ccccc2c1=O</smiles>

Fig 1. Structure of blue reaction product. 


\subsection{Validation of UV-Spectrophotometric Method}

Selectivity:

Using SCA selectivity was achieved measuring by normal spectrophotometry at $570 \mathrm{~nm}$ in $70 \mathrm{vol} . \%$ ethanol matrix.

(1) taurine / caffeine mixture
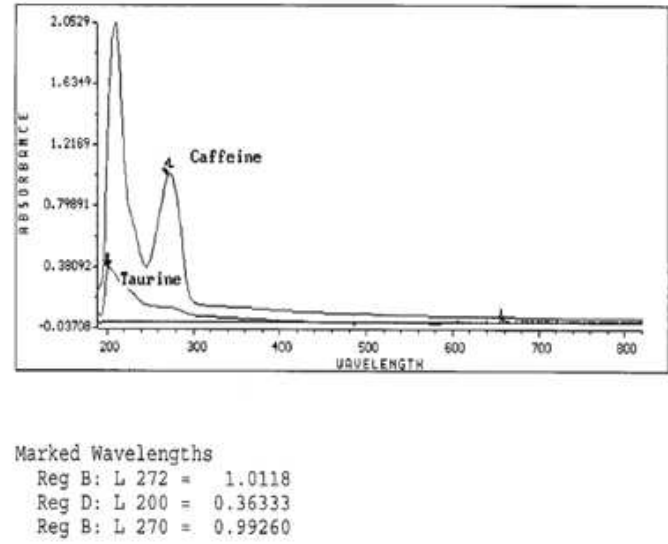

(2) taurine-ninhydrine color compound:

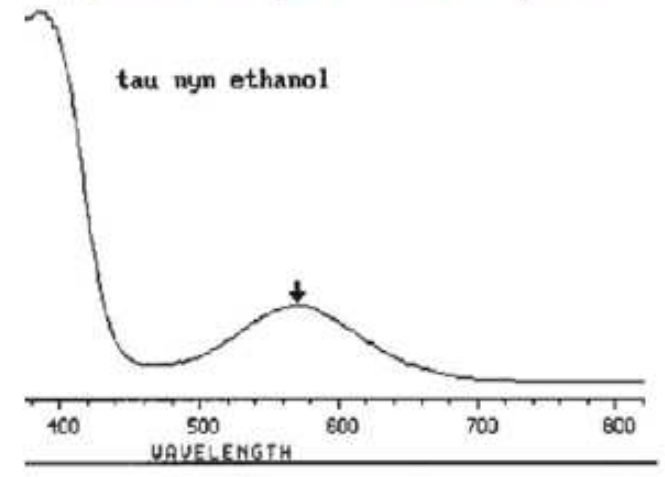

Marked wavelengths

Reg A: L $570=0.78914$

Fig 2. UV spectra of taurine / caffeine mixture (1) and taurine-ninhydrine color compound (2) in 70 vol. \% ethanol matrix.

\section{Precision:}

Six (6) equal solutions from homogenous samples containing $35 \mathrm{mg}$ taurine were analyzed by UV-spectrophotometric method. Standard deviation (SD = $0.00534 \mathrm{AU})$ and relative SD (RSD $=+/-1.44 \%)$ were found based on obtained absorption values. The results are presented on Table 2.

Table 2. Precision of samples containing taurine.

\begin{tabular}{lllll}
\hline Samples (n) & Obtained A (AU) & $\boldsymbol{X}_{\text {mean }}$ & SD & RSD (\%) \\
\hline 1 & 0.376 & & & \\
2 & 0.361 & & & \\
3 & 0.373 & 0.371 & 0.0053 & $\pm 1.44 \%$ \\
4 & 0.370 & & & \\
5 & 0.374 & & & \\
6 & 0.373 & & & \\
\hline
\end{tabular}

Accuracy:

Model mixtures of solutions containing 25, 35 and $50 \mathrm{mg}$ taurine in concentration ratio $50-150 \%$ of theoretical calculated quantity were prepared and analyzed three times each. The results are shown on Tables 3 . They are presented as $\%$ recovery. At fixed analytical parameters all studied combinations with ratio $25 / 35 / 50 \mathrm{mg}$ taurine : $50 \mathrm{mg}$ caffeine responds to ICH and Pharmacopoeia requirements about accuracy tolerance.

Table 3. Accuracy of model mixtures 1, 2 and 3 containing 25, 35 and $50 \mathrm{mg}$ taurine respectively.

\begin{tabular}{llll}
\hline $\begin{array}{l}\text { Model } \\
\text { mixtures (n) }\end{array}$ & $\begin{array}{l}\text { Putted amount } \\
\text { of taurine (g) }\end{array}$ & $\begin{array}{l}\text { Obtained results } \\
\text { for A (AU) }\end{array}$ & RSD (\%) \\
& & 0.183 & \\
1 & 0.025 & 0.180 & \pm 2.56 \\
& & 0.174 & \\
& & 0.202 & \pm 2.68 \\
2 & 0.035 & 0.213 & \\
& & 0.206 & \pm 0.55 \\
& & 0.370 & \\
\hline
\end{tabular}

Limit of detection:

$140 \mu \mathrm{g}$ for taurine, established on the base of ratio noise signal $-1: 3$. Results are shown on fig. 3 .

Limit of quantitation:

$1400 \mu \mathrm{g}$ for taurine, established on the base of ratio noise - signal - 1:10.

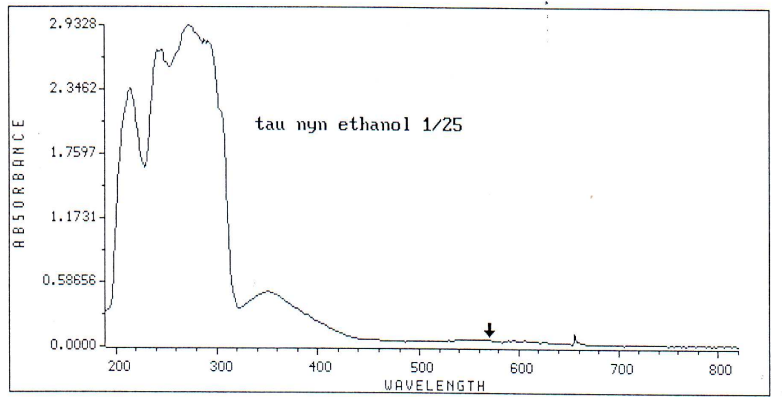

Marked Wavelengths

Reg A: L $570=0.06563$

Fig 3. UV-spectra of taurine-ninhydrin color compound in concentration ratio $1: 25 \mathrm{v} / \mathrm{v}$.

Linearity:

The analytical parameter linearity was studied in concentration ratio $0.1-0.004 \mathrm{~g}$. The accordance between the absorption, measured in absorption units (AU) and concentrations in $\mathrm{g} / \mathrm{ml}$ is proportional in the intervals. The correlation coefficients were found to be about $1-0.99692$ at $\mathrm{SD}=+/-0.02151 \mathrm{AU}, \mathrm{N}=7$ and $\mathrm{P}<0.0001$. Results for linearity of color compound in ethanol matrix are shown on table 4 . 
Table 4. Linearity parameters of taurine-ninhydrine color compound in 70 vol. \% ethanol matrix.

\begin{tabular}{lll}
\hline & \multicolumn{1}{c}{$\mathbf{Y}=\mathbf{A}+\mathbf{B} * \mathbf{X}$} & \\
Parameter & Value & Error \\
\hline $\mathrm{A}$ & 0.06108 & 0.01136 \\
$\mathrm{~B}$ & 72.20057 & 2.53899 \\
$R$ & $\mathrm{~N}$ & $\mathrm{P}$ \\
0.99692 & 7 & $<0.0001$ \\
\hline
\end{tabular}

\section{Conclusion}

The conditions of novel UV-spectrophotometry method for determination of taurine in supplement and drug energy mixtures were performed. The method was validated in complains with European Pharmacopoeia criteria and it distinctive properties are high selectivity, optimal values of analytical parameters and wide spectra of applications for purposes of pharmaceutical and toxicological practices.

\section{References}

[1] Jiao Yang, Cai-Rong Zhou, Xiao-Hua Shi, Determination and Correlation of the Solubility for Taurine in Water and Organic Solvent Systems, J. Chem. Eng. Data, 2010, 55 (7), Pages 2620-2623.

[2] Glutathione and Sulfur Amino Acids in Human Health and Disease, 2009, 22.6. Beneficial action of taurine, Pages 551, 553 - 56.

[3] H. Pasantes-Morales, A. Schousboe, Role of taurine in osmoregulation in brain cells: Mechanisms and functional implications, Amino Acids, 1997, Volume 12, Issue 3-4, Pages 281-292.

[4] Birdsall T.C., Therapeutic applications of taurine, Altern. Med. Rev., 1998, 3 (2), Pages 128-136.

[5] XiFeng Wang, DeFeng Chi, GuanMin Su, Lin Li, LiHua Shao, Determination of Taurine in Biological Samples by High-Performance Liquid Chromatography Using 4-Fluoro-7-Nitrobenzofurazan as a Derivatizing Agent, Biomedical and Environmental Sciences, October 2011, Volume 24, Issue 5, Pages 537-542.

[6] Yoshiyuki Sawabe, Takaomi Tagami, Katsuhiro Yamasaki, Determination of Taurine in Energy Drinks by HPLC Using a Pre-column Derivative, Journal of Health Science, 2008, Vol.54, No.6, Pages 661-664.

[7] Brad McConn, Determination of taurine in energy drinks by high-performance liquid chromatography, Concordia College, Journal of Analytical Chemistry, 2012, 3, 47-52.

[8] Aono Motomu, Soga Hideki, A Spectrophotometric Method for the Determination of Taurine in Nutritional Supplement Drinks, Scientific Reports of the Faculty of Agriculture, 2001,
Pages 109-117.

[9] O. Ferreiraa, M. V. Nunesa, E. Mendesa, F. Remiãob \& M. A. Ferreiraa, Development of An HPLC-UV Method for Determination of Taurine in Infant Formulae and Breast Milk, Journal of Liquid Chromatography \& Related Technologies, 1997, Volume 20, Issue 8, Pages 1269-1278.

[10] Zheng G., Lin S., Wei Sheng, Yan Jiu, Determination of taurine in foods by high performance liquid chromatography, Journal of Hygiene Research 1998, 27 (4), 266-8.

[11] Zhu Hui, Zhao Zhi-Hong, Shi Wen-Rong, Determination of Taurine in Food by Pre-column HPLC with Sulfonyl Chloride, Food Science, 2000, 21 (12), 116-11.

[12] Inoue H., Fukunaga K., Tsuruta Y., Determination of taurine in plasma by high-performance liquid chromatography using 4-(5,6-dimethoxy-2-phthalimidinyl)-2-methoxyphenylsulfon $\mathrm{yl}$ chloride as a fluorescent labeling reagent, Analytical Biochemistry, 2003, Volume 319, Issue 1, Pages 138-142.

[13] Zhi Chen, Bo Chen, Shouzhuo Yao, High-performance liquid chromatography/electrospray ionization-mass spectrometry for simultaneous determination of taurine and 10 water-soluble vitamins in multivitamin tablets, Analytica Chimica Acta, 2006, Volume 569, Issues 1-2, Pages 169-175.

[14] Aranda M., Morlock G., Simultaneous determination of riboflavin, pyridoxine, nicotinamide, caffeine and taurine in energy drinks by planar chromatography-multiple detection with confirmation by electrospray ionization mass spectrometry, Journal of Chromatography A, 2006, Volume 1131, Issues 1-2, Pages 253-260.

[15] Emilia Marchei, Manuela Pellegrini, Roberta Pacifici, Ilaria Palmi, Simona Pichini, Development and validation of a high-performance liquid chromatography-mass spectrometry assay for methylxanthines and taurine in dietary supplements, Journal of Pharmaceutical and Biomedical Analysis, 2005, Volume 37, Issue 3, Pages 499-507.

[16] V. Maslarska, J. Tencheva, O. Budevsky, Potentiometric analysis of mixtures of acids and bases, Der Chemica Sinica, 2011, 2 (6), 325-330.

[17] Maslarska V, Tencheva J and Budevsky O, New approach in the treatment of data from an acid-base potentiometric titration, I. Monocomponent systems of monofunctional acids and bases, Analytical and Bioanalytical Chemistry, $2003,375,(2), 217-222$.

[18] Maslarska V, Tencheva $\mathbf{J}$ and Budevsky O, New approach in the treatment of data from an acid-base potentiometric titration II. The Determination of Polyfunctional Acids and Bases, Chemical Analysis (Warsaw), 2005, 50 (5), 815-824.

[19] Carey B. Bottom, Samir S. Hanna, Donald J. Siehr, Mechanism of the ninhydrin reaction, Biochemical Education, January 1978, Volume 6, Issue 1, Pages 4-5. 